\title{
Symmetry energy in neutron star matter
}

\author{
C.O. Dorso \\ Instituto de Física de Buenos Aires, Pabellón I, \\ Ciudad Universitaria, 1428 Buenos Aires, Argentina. \\ G.A. Frank \\ Unidad de Investigación y Desarrollo de las Ingenierías, \\ Universidad Tecnológica Nacional, Facultad Regional Buenos Aires, \\ Av. Medrano 951, 1179 Buenos Aires, Argentina. \\ J.A. López \\ University of Texas at El Paso, El Paso, Texas 79968, U.S.A.
}

(Dated: September 11, 2018)

\begin{abstract}
We investigate the structure attained by neutron star matter with proton to neutron ratios ranging from $x=0.1$ to 0.5 , densities in the range of $0.02 \mathrm{fm}^{-3}<\rho<0.085 \mathrm{fm}^{-3}$, and temperatures $T<4 \mathrm{MeV}$. In particular we study the pasta shapes and the phase changes previously observed in nuclear matter, as well as the behavior of the symmetry energy. We corroborate the existence of homogeneous to non-homogeneous phase transitions, and the different values of the symmetry energy at different densities and temperatures.
\end{abstract}

PACS numbers: PACS 24.10.Lx, 02.70.Ns, 26.60.Gj, 21.30.Fe

\section{INTRODUCTION}

Non-pulsar neutron stars are expected to cool down mainly through neutrino emission. Such emission depends initially on the convective motion of the neutron star matter, which is dictated by its equation of state [1, its dependence on isospin content, and by the structure of the neutron star crust which controls the opacity of the medium to the neutrino flow 14. This underlines the importance of studying both the dependence of the equation of state (EOS) of neutron star matter on isospin content (i.e. the symmetry energy, $E_{\text {sym }}$ ), and the structure of the neutron star crust at saturation and subsaturation densities, low temperatures and with varying isospin content.

The connection between the structure of a nuclear medium and the value of its symmetry energy was established experimentally in low-energy nuclear reactions at the Texas A\&M Cyclotron [3 5. These investigations showed that $E_{\text {sym }}$ is affected by the formation of clusters; such dependence on clustering was later corroborated by a calculation of $E_{\text {sym }}$ in clustered media [29, while non clustering mean-field theories failed to yield the asymptotic limit of $E_{\text {sym }}$ at small densities [7.

In a previous study, the structure and symmetry energy of nuclear matter (NM), a system closely related to neutron star matter (NSM), was studied 30 (NM is a conglomerate of protons and neutrons in which proton-proton Coulomb interaction is disregarded. NSM is NM with proton-proton Coulomb term activated, embedded in an electron cloud (the system as a whole is neutral). This electron cloud renders the Coulomb interaction short ranged, and then, the energy and entropy of NSM is additive). Such study focused on the structure of neutron-rich nuclear matter and, in particular, on the behavior of the symmetry energy on those structures. It was found, first, that the so-called "pasta" structures exist in neutron-rich nuclear matter and that they exhibit the crystal-to-solid-to-liquid phase transitions found in Ref. [12] for isospin-symmetric NM. And, second, that the symmetry energy depends on the morphology of the pasta and on its phase transitions.

This motivates the questions of the present study: how does the topology of the NSM evolves as temperature is lowered in terms of its isospin asymmetry? Do NSM exhibit phase transitions similar to those observed in NM? And, how does the symmetry energy depend on these structures and phase changes? The importance of these questions lies on the potentially large impact these effects can have on neutrino cooling and in the synthesis of heavy nuclei in neutron star mergers [22].

These questions can be addressed from a theoretical point of view using a model capable of forming the pasta structures, undergoing phase transitions, while allowing the evaluation of $E_{\text {sym }}$ in these cold and inhomogeneous systems. Although several models exist, not all of them allow the simultaneous calculation of the structures, phase transitions and of the symmetry energy simultaneously.

Nuclear pastas are spatial arrangement of protons and neutrons, theorized to exist in neutron star crusts 8. To obtain these structures, nuclei in the NSM should reach a free energy minima. In the original studies of the 1980s the energy minima were determined using static methods such as the liquid drop model [8, 9], mean field theo- 
ries [10] and Thomas-Fermi models [1]. These methods, however, usually work only at zero temperature, locate only the "traditional" global minima and tend to miss the "non-traditional" local minima of energy barriers; to gain access to the complete set of structures (traditional and non-traditional) temperature-dependent dynamical models are needed.

Dynamical studies of the pastas have been performed with quantum molecular dynamics [16, 17, 26] and classical potential models [2, 6, 25]. But to properly obtain the pasta structures, including the non-traditional phases, it is best to use models that can identify local minima by cooling, such as the classical molecular dynamics model (CMD) [12].

The CMD has been used, for instance, to calculate $E_{\text {sym }}$ in clustered media [29], and to study the pasta structures and phase changes in NM, as well as the symmetry energy in the different pasta structures and in all of its phases [12].

In this work we extend the study of nuclear matter of Ref. [30] to the realm of neutron star matter. We use CMD to study, first, the existence of pasta-like structures and their possible phase changes at saturation and sub-saturation densities, low temperatures, and proton fractions in the range of $10 \%$ to $50 \%$. Second, we compute the behavior of $E_{\text {sym }}$ in the different structures found.

\section{CLASSICAL MOLECULAR DYNAMICS}

This work uses classical molecular dynamics, a wellestablished computational technique that has been used to determine the pasta structures that form in NM and NSM 2, 6, 12,14. CMD represents nucleons as classical particles interacting through pair potentials and calculates their dynamics by solving their equations of motion numerically. CMD has several advantages that have been presented elsewhere [2, 6, 12, and its validity on nuclear systems at the range of temperatures and densities achieved in intermediate-energy nuclear reactions has been discussed before [13].

In this section we introduce the nucleon-nucleon potentials used in CMD along with the tools used in the study of the resulting structures, namely, the Minkowski functionals and the symmetry energy. A more thorough description of these tools can be found in [30.

\section{A. Potentials}

Neutron star matter is composed of protons, neutrons and electrons, CMD uses $p p, n n$ and $n p$ potentials, as

\begin{tabular}{lcrc}
\hline \hline Parameter & Pandharipande & New Medium & Units \\
\hline$V_{r}$ & 3088.118 & 3097.0 & $\mathrm{MeV}$ \\
$V_{a}$ & 2666.647 & 2696.0 & $\mathrm{MeV}$ \\
$V_{0}$ & 373.118 & 379.5 & $\mathrm{MeV}$ \\
$\mu_{r}$ & 1.7468 & 1.648 & $\mathrm{fm}^{-1}$ \\
$\mu_{a}$ & 1.6000 & 1.528 & $\mathrm{fm}^{-1}$ \\
$\mu_{0}$ & 1.5000 & 1.628 & $\mathrm{fm}^{-1}$ \\
$r_{c}$ & 5.4 & $5.4 / 20$ & $\mathrm{fm}$ \\
\hline \hline
\end{tabular}

TABLE I. Parameter set for the CMD computations. The values used in this work correspond to the New Medium Model.

well as an screening potential to mimic the effect of the electron gas; these potentials are now described in turn.

\section{1. nucleon-nucleon potentials}

In the CMD model nucleons interact through the Pandharipande (Medium) potentials. These potentials attain a binding energy $E\left(\rho_{0}\right)=-16 \mathrm{MeV} /$ nucleon and a compressibility of about $250 \mathrm{MeV}$. The corresponding mathematical expressions are

$$
\begin{aligned}
& V_{n p}(r)=\frac{V_{r}}{r} e^{-\mu_{r} r}-\frac{V_{r}}{r_{c}} e^{-\mu_{r} r_{c}}-\frac{V_{a}}{r} e^{-\mu_{a} r}+\frac{V_{a}}{r_{c}} e^{-\mu_{a} r_{c}} \\
& V_{n n}(r)=\frac{V_{0}}{r} e^{-\mu_{0} r}-\frac{V_{0}}{r_{c}} e^{-\mu_{0} r_{c}}
\end{aligned}
$$

where $r_{c}$ is the cutoff radius after which the potentials are set to zero. Although the parameters $\mu_{r}, \mu_{a}, \mu_{0}$ and $V_{r}, V_{a}, V_{0}$ were first set by Pandharipande for cold nuclear matter [23, a recent improvement 22], here named New Medium, reproduces the cold nuclear matter binding energies more accurately and, thus, is used in this work. The corresponding values are summarized in TableI. Figs. 1a and $1 \mathrm{~b}$ contrasts these potentials with those of Pandharipande Medium potentials.

\section{The Coulomb potential}

As the neutron star matter contains protons and neutrons embedded in an electron gas, it is necessary to include the Coulomb effect of the gas. The main effect of the electron cloud is to introduce an screening effect on the Coulomb potential of the protons. The screened Coulomb potential may be expressed through the Thomas-Fermi expression [2, 25, 26]

$$
V_{t f}=\frac{q^{2}}{r} e^{-r / \lambda},
$$

where the screening length is $\lambda=20 \mathrm{fm}$, and the cutoff distance for $V_{t f}$ is $20 \mathrm{fm}$. The values were selected to be 


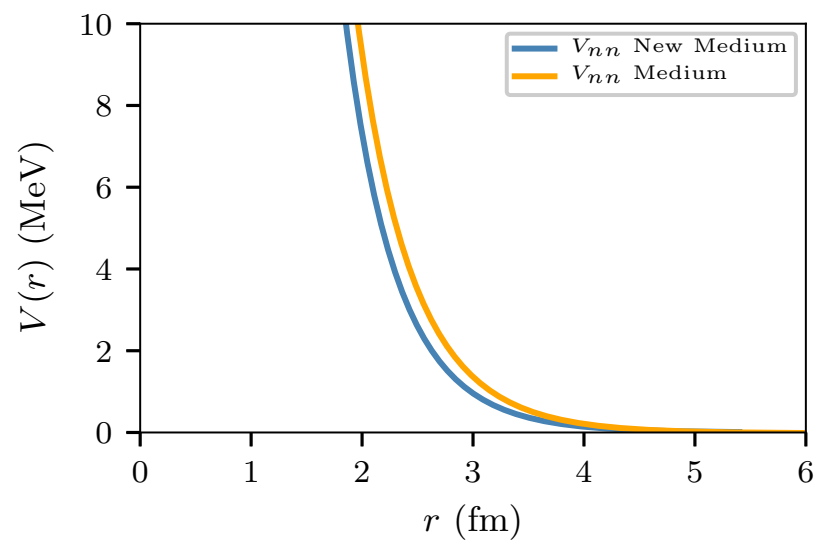

(a) $V_{n n}$

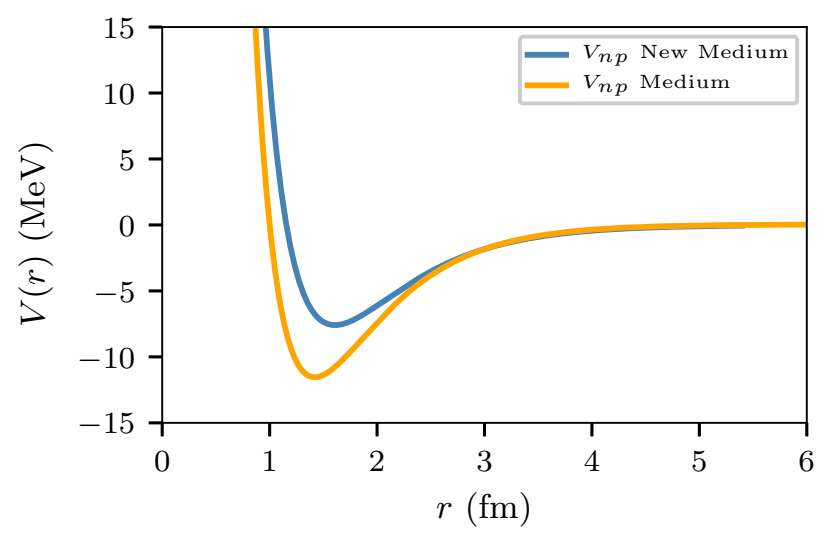

(b) $V_{n p}$

FIG. 1. (color online) Potential profiles for interacting nucleons (in MeV). The orange curves correspond to the Pandharipande Medium model. The blue curves correspond to the improved New Medium model (see text for details).

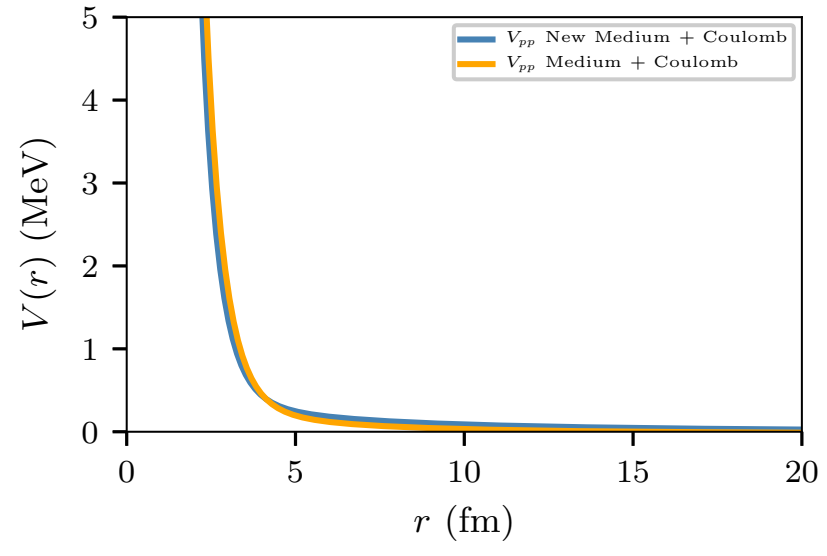

FIG. 2. (color online) Potential profiles for interacting protons (in $\mathrm{MeV}$ ). The orange curve corresponds to the Pandharipande Medium model embedded in the Thomas-Fermi potential (see Eq. (2)). The blue curve corresponds to the improved New Medium model embedded in the Thomas-Fermi potential (see Eq. 22).

long enough to reproduce the density fluctuations in the cell size used (see Section IIC] 28] .

Figs. 1 shows the interaction potentials between nucleons, without the existence of the surrounding electron gas, while Fig. 2 shows proton-proton complete potential (including the Coulomb screening).

It might be appropriate at this point to emphasize that the exponential cut-off renders the Coulomb effective interaction, short ranged. In this way the energy and the entropy is additive (i.e. energy scales with the number of particles).

\section{B. Tools}

To characterize the pasta here we use the Minkowski functionals [27], which allow us to measure the size, shape and connectivity of spatial structures formed by the nucleons. In this section we also present the procedure used for the evaluation of the symmetry energy from CMD data [13, 29].

\section{Minkowski functionals}

The Minkowski functionals 27] are shape descriptors that have been used extensively in the pasta literature (see e.g. 6, 18, 20]). For three-dimensional bodies, these functionals are the volume, surface area, Euler characteristic $(\chi)$, and integral mean curvature (B). The Euler characteristic can be interpreted as

$$
\chi=\text { isolated regions }+ \text { cavities }- \text { tunnels }
$$

while $B$ is a measure of the curvature of the surface of a given structure. In Ref. 6 it was found that the pasta structures can be classified according to Table II. i.e. a characterizing connection exists between the different structures with the curvature (B) and the Euler characteristic; the signs of these functionals are also correlated with the pasta phases [21]. In our case, to calculate the Minkowski functionals on pasta structures produced by CMD, the structures must be "voxelized", i.e., represented by "voxels", or regular units of volume; the appendix of [30] presents the procedure used.

\section{Symmetry energy}

The evaluation of the symmetry energy follows the procedure introduced in Refs. [13, 29]. The symmetry energy 


\begin{tabular}{l|c|c|c}
\hline \hline & $\mathrm{B}<0$ & $\mathrm{~B} \sim 0$ & $\mathrm{~B}>0$ \\
\hline$\chi>0$ & Anti-Gnocchi & & Gnocchi \\
$\chi \sim 0$ & Anti-Spaghetti & Lasagna & Spaghetti \\
$\chi<0$ & Anti-Jungle Gym & & Jungle Gym \\
\hline \hline
\end{tabular}

TABLE II. Integral mean curvature and Euler characteristic values for pasta shapes. The "anti" prefix means the inverted situation between occupied and empty regions. The "jungle gym" stands for a 3D rectangular trellis.

is defined as

$$
E_{\text {sym }}(\rho, T)=\left.\frac{1}{2 !} \frac{\partial^{2} E(\rho, T, \alpha)}{\partial \alpha^{2}}\right|_{\alpha=0}
$$

with $\alpha=(N-Z) /(N+Z)=1-2 x$. Using the CMD results of the internal energy $E(\rho, T, x)$ it is possible to construct a continuous function by fitting (i.e. least squares estimation) the values of $E(T, \rho, \alpha)$ for each $T$ and $\alpha$ with an expression of the type

$$
E(T, \rho, \alpha)=\sum_{i=0}^{3} E_{i}(T, \alpha) \rho^{i}
$$

The $\alpha$ dependence of the coefficients $E_{i}(T, \alpha)$ can be extracted from the CMD data calculated at various values of $\alpha$, and assuming an $\alpha$ dependence of the type

$$
E_{i}(T, \alpha)=E_{i 0}(T)+E_{i 2}(T) \alpha^{2}+E_{i 4}(T) \alpha^{4}
$$

with odd terms in $\alpha$ not included to respect the isospin symmetry of the strong force. The symmetry energy is then given by

$$
\begin{aligned}
E_{\text {sym }}(T, \rho) & =E_{02}(T)+E_{12}(T) \rho+ \\
& +E_{22}(T) \rho^{2}+E_{32}(T) \rho^{3}
\end{aligned}
$$

with the coefficients $E_{i j}(T)$ obtained from the fit of the CMD data.

\section{Molecular dynamics simulation of neutron star matter}

In order to mimic (asymmetric) neutron star matter, the LAMMPS CMD code 24] was fitted with the potentials mentioned in Sections IIA1 and IIA2, Graphics Processing Units (GPU) were used to carry out the computations. We tracked the evolution of systems with $A=4000$ nucleons situated in a cubic cell under periodic boundary conditions. The simulated isospin content was $x=z / A=0.1,0.2,0.3,0.4$, and 0.5 . The densities were varied between $0.02 \mathrm{fm}^{-3}$ to $0.085 \mathrm{fm}^{-3}$. The temperature was controlled with a Nosé-Hoover thermostat slowly varying from $T=4 \mathrm{MeV}$ down to $0.2 \mathrm{MeV}$ $(\Delta T<0.1 \%)$. After placing the nucleons at random, but with a minimum inter-particle distance of $0.01 \mathrm{fm}$, the nucleons were endowed with velocities according to a Maxwell-Boltzmann distribution to correspond to a desired temperature, and the equations of motion were solved to mimic the evolution of the system. The nucleon positions, momenta, energy per nucleon, pressure, temperature, and density, were stored at fixed time-steps.

\section{RESULTS FOR NSM PASTA}

With the tools presented in the previous section we now analyze the pasta structures to determine whether the phase transitions obtained in nuclear matter 30 survive in neutron star matter, and what is the behavior of the symmetry energy within the pasta.

\section{A. Symmetric neutron star matter}

We first study the case of symmetric neutron star matter, i.e with $x=z / A=0.5$, focusing on the caloric curve and the binding energy.

\section{The caloric curve}

For starters we calculate the internal energy of symmetric neutron star matter. This involves averaging the kinetic and potential energy of each nucleon in the system.

To detect possible phase changes it is customary to calculate the caloric curve, which plots the relationship between the internal energy and the temperature. This curve indicates the amount of heating produced as a function of the energy added to the system. During a phase transition, however, the addition of energy does not produce a change in temperature because the energy is not used to heat the system but to break bonds melting the system or liberating liquid molecules. Thus, changes in the slope of the $E-T$ curve can be used as indicators of phase transitions.

In our case it is convenient to remember that, as seen in Ref. [30], nuclear matter maintains a liquid-like structure for temperatures larger than about $2.0 \mathrm{MeV}$, temperature at which it freezes into a pasta structure composed of nucleons arranged amorphously (still like a liquid). And at around $T=0.5 \mathrm{MeV}$ it transforms into a pasta with nucleons frozen much like in crystalline structures.

For neutron star matter the results are somewhat different. Figure 3 presents the case of $\rho=0.05 \mathrm{fm}^{-3}$, 
and Figure 4 shows the curves for densities $\rho=0.02$, $0.05,0.10$ and $0.16 \mathrm{fm}^{-3}$ in the extended temperature range of 0.2 to $4 \mathrm{MeV}$. In both figures the total number of nucleons in the primary cell was $N=4000$ interacting through the New Medium model (c.f. Section II).

As it can be seen more clearly in Fig. 3, the slope of the internal energy exhibits a change at around $T \simeq 1.5 \mathrm{MeV}$, and a sharper change at $T \simeq 0.5 \mathrm{MeV}$. Although these jumps are not as pronounced as those found for NM, they happen at around the same temperatures and can be taken as the same type of transitions.

We claim that the smooth change at $T \simeq 1.5 \mathrm{MeV}$ signals the onset of the topological phase transition, (i.e. a bubble appears). A difference with NM is that this change in slope appears more pronounced for smaller densities $\left(\rho \leq 0.05 \mathrm{fm}^{-3}\right)$, and tends to disappear for larger densities. As this result is different than the NM case, we believe it is due to the presence of the electron gas.

On the other hand, the sharper jump observed at $T \simeq 0.5 \mathrm{MeV}$ both in Figs. 3 and 4 is maintained throughout the density range studied and, in fact, becomes more pronounced for larger densities. We believe this discontinuity in the derivative of the $E-T$ curve signals the amorphous pasta-crystalline pasta change already identified in Ref. [30] for NM with the Pandharipande Medium model.

The noticeable differences between the caloric curve of nuclear matter and that of neutron star matter are shown in Fig. 5. The figure compares the NSM results (continuous lines) against the NM curves (dashed lines) for the cases of $\rho=0.05,0.06,0.07$ and $0.085 \mathrm{fm}^{-3}$ and temperatures from 0.2 to $4 \mathrm{MeV}$. The NSM lines remain separated and parallel throughout the range of explored temperatures, whereas the NM curves merge with one another at low temperatures. Noteworthy, the NM highest density curve $\left(\rho=0.085 \mathrm{fm}^{-3}\right)$ corresponds to the lowest energy, while the opposite is true for NSM where the higher density curve has the higher energy of all the curves shown.

\section{The energy}

When plotted against the density, the energy can be a useful indicator of the state of the system. As seen in the case of NM [30, the $E-\rho$ curve can signal the saturation density (i.e. the minimum of the curve), bound and unbound states (positive or negative energies), the range of densities of the liquid phase (span of the " $\bigcup$ " shape), and changes of phase, among others. For the case of NSM, however, the situation is totally different.

Fig. 6 shows the energy as a function of the average

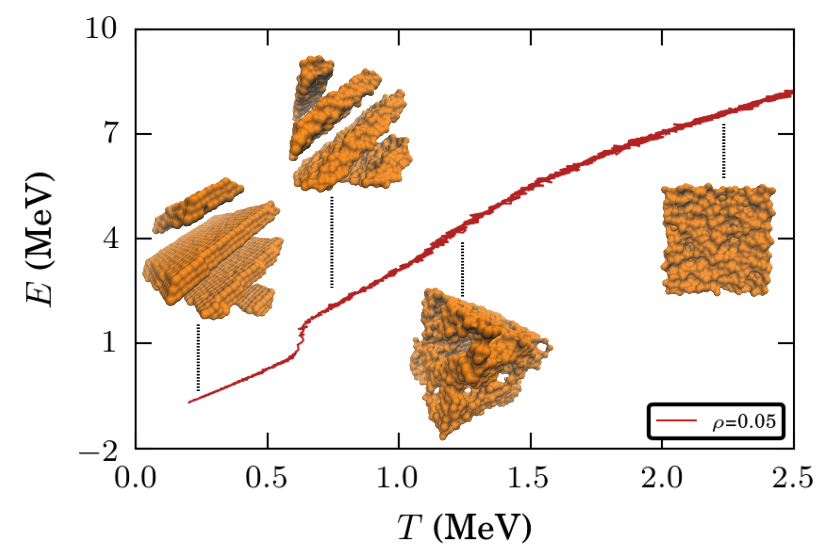

FIG. 3. (color online) Internal energy per nucleon for symmetric neutron star matter $(x=0.5)$ as a function of the bath temperature, for $\rho=0.05 \mathrm{fm}^{-3}$.

density at $T=0.1,0.2,1.0$ and $2.0 \mathrm{MeV}$, as obtained from the CMD simulations; these results, interestingly, differ substantially from the behavior exhibited by symmetric nuclear matter. As shown in the four lines, there is no absolute minima and, thus, NSM at these temperatures and densities does not have an equilibrium point nor a saturation density; this is different than the $U$ shape that what was found for NM. Likewise, at a difference from NM, for most of the densities the NSM system is unbound, except for $T \leq 1.0 \mathrm{MeV}$ and $\rho \leq 0.05 \mathrm{fm}^{-3}$.

The difference between nuclear matter and neutron star matter is that for NM there is no embedding electron gas, whereas for NSM the effect of the cloud of electrons introduces the short range Coulomb screening. It is quite interesting that because the exponential cut-off is of the order of $20 \mathrm{fm}$, small aggregates of nucleons resemble nuclei quite closely.

\section{The Minkowski functionals}

A first insight to the topology associated to the structures formed may be given by the Minkowski functionals. As explained before, the Minkowski functionals [27] use the volume, surface area, Euler characteristic $\chi$, and integral mean curvature B to characterize the size, shape and connectivity of spatial structures formed by the nucleons. The calculation of the Minkowski functionals requires the binning of nucleons into "voxels" which have to be of the proper size (see, e.g., the Appendix of Ref. [30 for the procedure to use). In this work cubic voxels of $d=2.35 \mathrm{fm}$ of length were used, and the Euler 


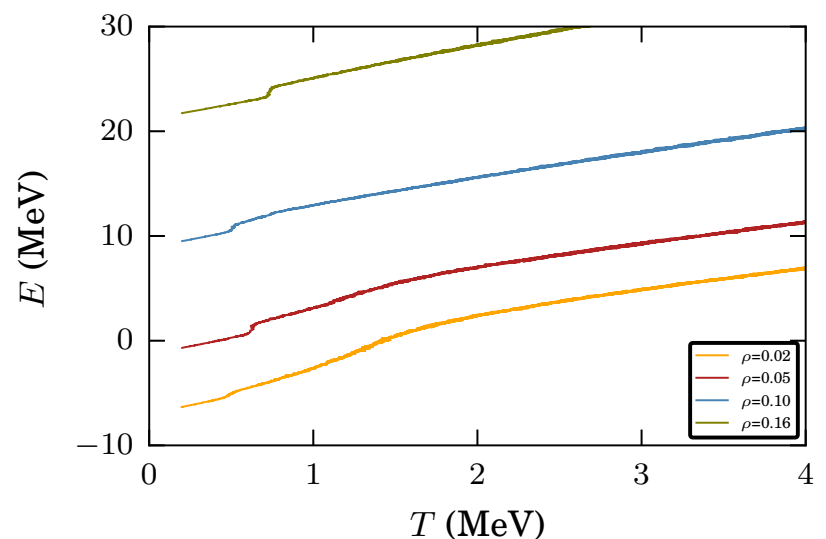

FIG. 4. (color online) Internal energy per nucleon for symmetric neutron star matter $(x=0.5)$ as a function of the bath temperature. The mean density for each profile is indicated in the insert.

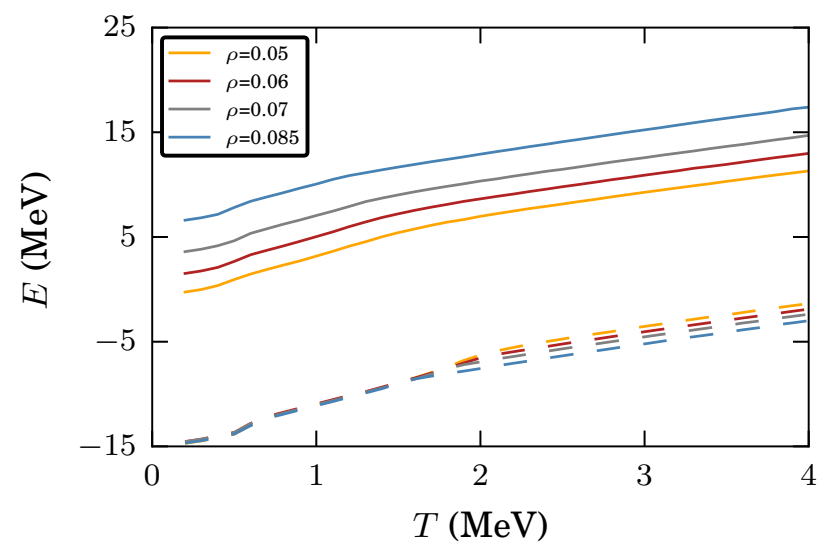

FIG. 5. (color online) Internal energy per nucleon for symmetric neutron star matter $(x=0.5)$ as a function of temperature. The mean density for each profile is indicated in the inset in $\mathrm{fm}^{-3}$. The dashed lines correspond to the Pandharipande Medium model (nuclear matter), while the continuous lines correspond to the New Medium potential with the screened Coulomb potential.

functional $\chi$ was computed according to Eq. (3).

Figure 7 shows the Euler functional $\chi$ as a function of the the temperature. Although the $\chi$ may somewhat depend on the binning distance of the simulation cell, the exhibited patterns are qualitatively meaningful. Several features are worth mentioning.

$\chi$ has a clear change of behavior at $T \simeq 1 \mathrm{MeV}$. Although this happens at a lower temperature than its NM counterpart (see Fig. 16 in Ref. 30]), it also appears to be associated to the early stage of the pasta formation. Furthermore, the $\chi$ values for the examined densities

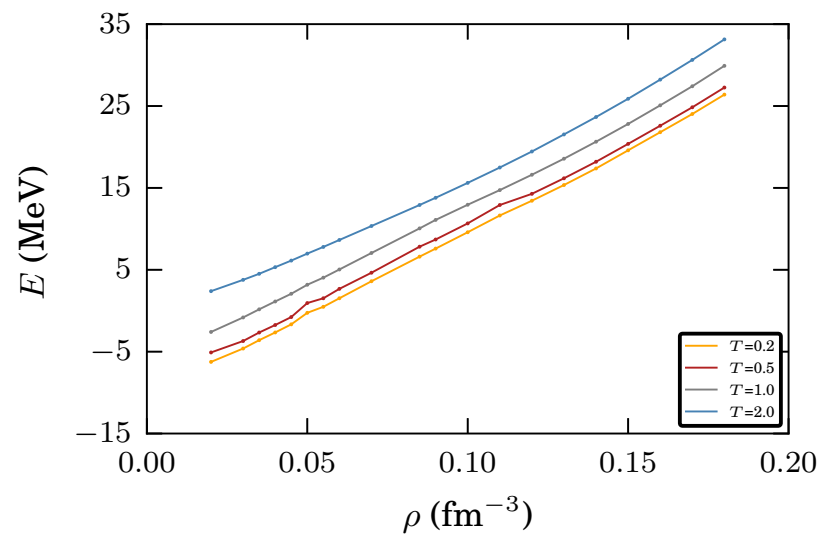

FIG. 6. (color online) Internal energy per nucleon for symmetric neutron star matter $(x=0.5)$ as a function of the mean density at fixed temperatures. The corresponding bath temperature for each is indicated in the insert.

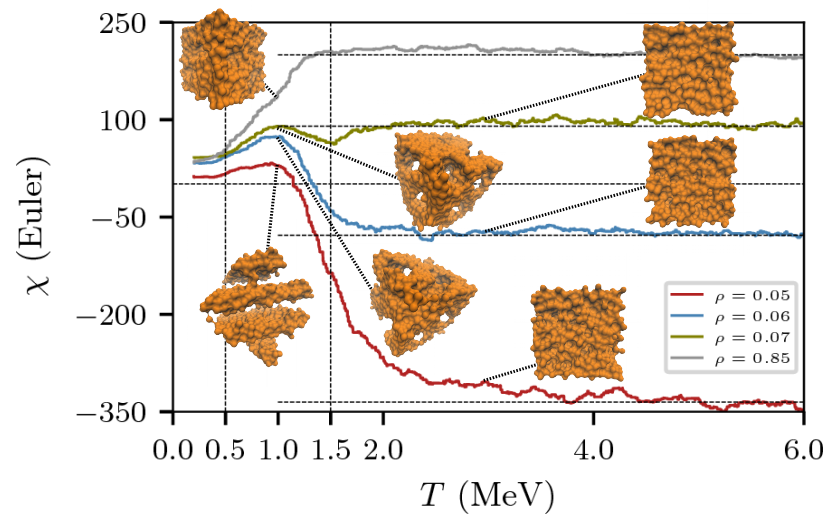

FIG. 7. (color online) The Euler functional $\chi$ obtained for isospin symmetric $(x=0.5)$ NSM systems as a function of temperature. The systems had the densities shown in the inset $\left(\right.$ in $\mathrm{fm}^{-3}$ ) and were composed by $N=4000$ nucleons interacting through the New Medium model. The corresponding binning distance is $d=2.35 \mathrm{fm}$. The data has been smoothed with a moving average procedure.

almost join into a single pattern for $T \leq 0.5 \mathrm{MeV}$, much like those of $\mathrm{NM}$ at the same temperature but less pronounced; this could also be related to the change of slope found in the caloric curve at $T \simeq 0.5 \mathrm{MeV}$.

A look at the sign of $\chi$ can yield information about the morphology of the structure. Figure 7 indicates that the lower density systems attain negative values of $\chi$ at, say, $T>1 \mathrm{MeV}$. Since values of $\chi<0$ corresponds to cases where the number of tunnels overcome the number of voids and isolated regions, see Eq. (3), it seems that 


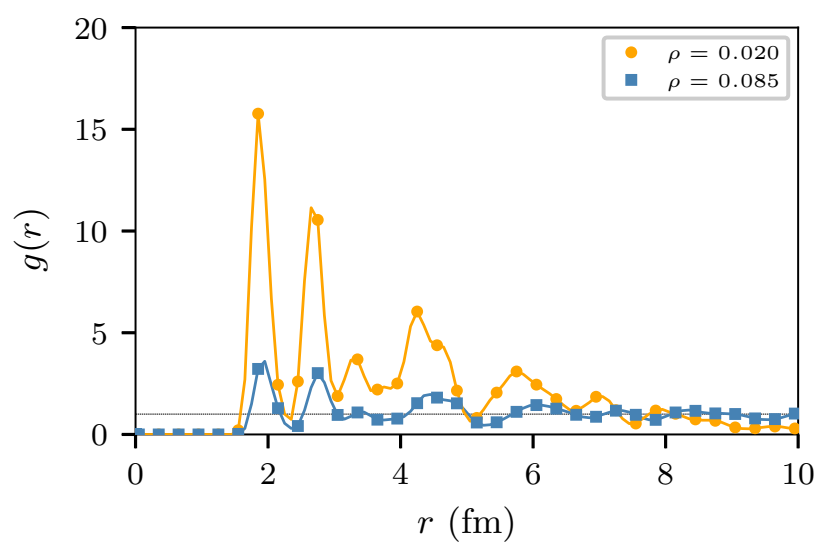

FIG. 8. (color online) Radial distribution function $g(r)$ for nucleons corresponding to a symmetric neutron star matter system of $N=4000$ nucleons and $T=0.2 \mathrm{MeV}$. The system mean density is indicated in the inset in $\mathrm{fm}^{-3}$. The horizontal line at 1 corresponds to the asymptotic limit expected for infinite systems.

low density configurations tend to be more cavity-like (more tunnels), but as density increases tunnels fill up yielding more compact structures.

In summary, and comparing to NM, the introduction of the coulomb screened potential appears to smooth out the phase transitions. The Euler functional $\chi$ experiences a somewhat smooth change along $1-2 \mathrm{MeV}$, in correspondence with the energy changes mentioned in Section III A 1. Thus, the "pasta" forming process may be located at this temperature range, in a similar fashion as in nuclear matter systems (see Ref. 30]). At temperatures below $T \simeq 0.5 \mathrm{MeV}$ the (inner) nucleons in the "pasta" structure freeze into a solid state.

\section{The radial distribution function}

The radial distribution function $g(r)$ can be used to explore the phase transformations detected by the caloric curve by looking at the average distribution of nearest neighbors. Fig. 8 shows the $g(r)$ for two systems with $N=4000$ nucleons interacting through the New Medium model at two sub-saturation densities. The $\rho=0.02$ profile resembles a more regularly distributed system, with more pronounced nearest neighbor peaks, as compared to the $\rho=0.085$ profile. Undoubtedly, the lower density case corresponds to a more crystalline phase than the high density which appears less structured and, looking at Figure 5, we see that the highest internal energy is associated to a more regular distributions of nucleons within the pasta regime.

The correlation of $g(r)$ to the pasta morphology can be observed by plotting the spatial distribution of the protons. Fig. 9 shows the proton arrangements for three density situations. It can be checked from Fig. 9c that the $\rho=0.085$ situation is highly homogeneous inside the occupied regions, although hollow spaces (of protons) exist. These occupied regions split into smaller pieces, according to Figs. 9b and 9a, forming lasagnas and gnocchis, respectively. Thus, as the density diminishes (and the structures break into smaller pieces), the internal energy decreases (see fig. 6).

The connection to $g(r)$ is as follows. At the lowest explored density $\left(\rho=0.02 \mathrm{fm}^{-3}\right)$ of Figure $8 g(r)$ shows sharp peaks at $r \leq 6 \mathrm{fm}^{-3}$ and almost no correlation $(g(r) \approx 0)$ for $r \geq 8 \mathrm{fm}^{-3}$. This indicates that strong correlations exist between neighbors belonging to the same gnocchi, and no correlation at large separation distances between gnocchis (see Fig. 9a). For the larger density, $\rho=0.085 \mathrm{fm}^{-3}, g(r)$ tends to 1 as expected for homogeneous systems (c.f. 9c).

We may summarize Section IIIA as follows. The internal energy for symmetric neutron star matter increases monotonically for either increasing temperatures and densities; it does not appear to have a saturation point. Since these effects do not occur for nuclear matter, it can be said that the Coulomb potential is responsible for biasing the energy upward, as can be seen clearly by comparing Fig. 4 to the nuclear matter case. Likewise, the introduction of the Coulomb potential affects the system morphology; as the density diminishes, the pasta structures split into smaller structures.

\section{B. Non-symmetric neutron star matter}

\section{The internal energy}

As a second step in our study of NSM we explored the behavior of the internal energy in systems with decreasing fractions of protons. We investigated fractions from $x=0.1,0.2,0.3$ and 0.4 to mimic the proton fractions appearing in neutron crusts. All simulations consist of systems with $N=4000$ nucleons under periodic boundary conditions and interacting through the New Medium potential.

Figures $10 \mathrm{a}$ and $10 \mathrm{~b}$ show the isothermal energies as a function of $x$ for systems with densities $\rho=0.04 \mathrm{fm}^{-3}$ and $\rho=0.085 \mathrm{fm}^{-3}$. Each of the curves exhibits a $\cup$ shape indicating the existence of a minimum of the energy at a certain value of $x$. At $\rho=0.04 \mathrm{fm}^{-3}$ the observed minima appears to shift from $x=0.4$ at $T=0.2 \mathrm{MeV}$ to $x=0.3$ at $T=2.0 \mathrm{MeV}$, while at $\rho=0.085 \mathrm{fm}^{-3}$ the minima remain at $x=0.3$ at all temperatures. This finding indicates that systems with the freedom of exchanging their content of neutrons and protons would favor a specific isospin ratio $x$ depending 


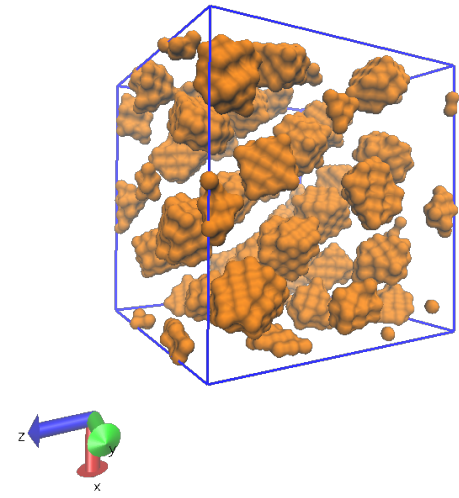

(a) $\rho=0.02$

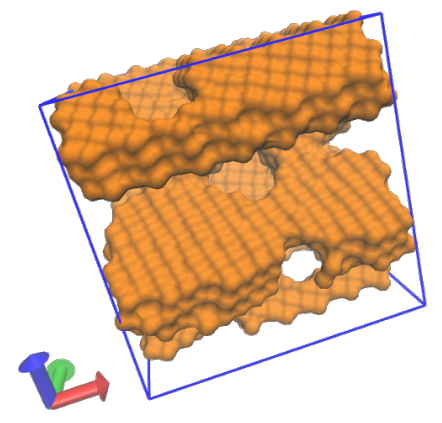

(b) $\rho=0.04$

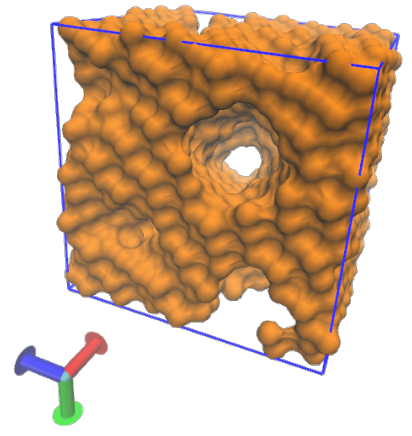

(c) $\rho=0.085$

FIG. 9. (color online) Surface representation for the protons at the indicated densities. The snapshot was taken at $T=0.2$ and $x=0.5$ for a system of 4000 nucleons.

on the local temperature and density of the system.

Complementary information can be obtained from the energy dependence of the energy isotherms. Figure 11 shows the corresponding curves for $x=0.2,0.4$ and 0.5 . It is interesting to notice that while the $x=0.4$ and 0.5 curves appear to have similar monotonically decreasing behaviors at all densities, the $x=0.2$ case deviates at low densities. As we will see next, this is due to the $\cup$ shape $x$ dependence of the energy seen in Figures 10a and $10 \mathrm{~b}$.

Specifically, Panel 11a shows the $T=0.2 \mathrm{MeV}$ case and, if focusing at $\rho=0.04 \mathrm{fm}^{-3}$, we see that $E(x=0.4)<E(x=0.2) \approx E(x=0.5)$, as can be expected by looking at the points at $x=0.2,0.4$ and 0.5 of the $T=0.2 \mathrm{MeV}$ isotherm in Figure 10a. Similarly, Panel $11 \mathrm{~b}$ can be understood by comparing to the $T=1.0 \mathrm{MeV}$ isotherm in Figure $10 \mathrm{~b}$. One can see, then, that the diverging behavior of the $x=0.2$ energy-density curves at low densities in Figure 11 is simply explained by the $\cup$ dependence of the energy on the isospin content $x$.

\section{The associated topology}

Following the footsteps of the case of symmetric NSM, we proceed to examine the Euler functionals $\chi$ and the radial distribution functions $g(r)$ for the non-symmetric systems. Fig. 12 shows the $\chi$ for four representative proton fractions at the densities $\rho=0.04 \mathrm{fm}^{-3}$ and $\rho=0.085 \mathrm{fm}^{-3}$.
In general terms, $\chi$ indicates changes of structure at around $T \approx 1 \mathrm{MeV}$. Figure $12 \mathrm{a}$ shows that at the lower density $\left(\rho=0.04 \mathrm{fm}^{-3}\right)$ and at temperatures hotter than $T \geq 2 \mathrm{MeV}$, the system appears dominated by voids and tunnels independent of the isospin content, while at lower temperatures $(T \leq 1 \mathrm{MeV})$ more compact objects appear at all $x$, except for $x=0.2$ which disperses even more. Figure $12 \mathrm{~b}$ shows that at the higher density $\left(\rho=0.085 \mathrm{fm}^{-3}\right)$ the system attains a more compact structure at all isospin contents and for $T \geq 1 \mathrm{MeV}$, becoming less pronounced at lower temperatures $(T \leq 1$ $\mathrm{MeV})$.

Figure 13 shows the space position of the protons for the cases of $x=0.2$ (a), $x=0.4$ (b), and $x=0.5$ (c) at $T=0.2$ and $\rho=0.085 \mathrm{fm}^{-3}$. Although not directly comparable to the values of $\chi$ obtained in Figure $12 \mathrm{~b}$ (recall that the computation of $\chi$ includes both neutrons and protons), the three structures in Figures 13 correspond to the left end points $(T=0.2$ $\mathrm{MeV}$ ) of the $\chi$ curves in Figure $12 \mathrm{~b}$ notice that no major changes in the percentage of void to filled volumes are observed as it is difficult to appreciate such changes in compact structures.

At lower densities, however, the situation is different. Figure 14 shows the protons for the cases of $x=0.1$, $0.2,0.3,0.4,0.45$ and 0.5 at $T=0.2 \mathrm{MeV}$ and $\rho=0.04$ $\mathrm{fm}^{-3}$. Comparing Figures $14 \mathrm{~b}$, $14 \mathrm{~d}$ and $14 \mathrm{f}$ to the the left end $(T=0.2 \mathrm{MeV})$ values of $\chi$ obtained in Figure 12 it is easy to see the how the voids and tunnels decrease as $x$ goes from 0.2 to 0.5 . 


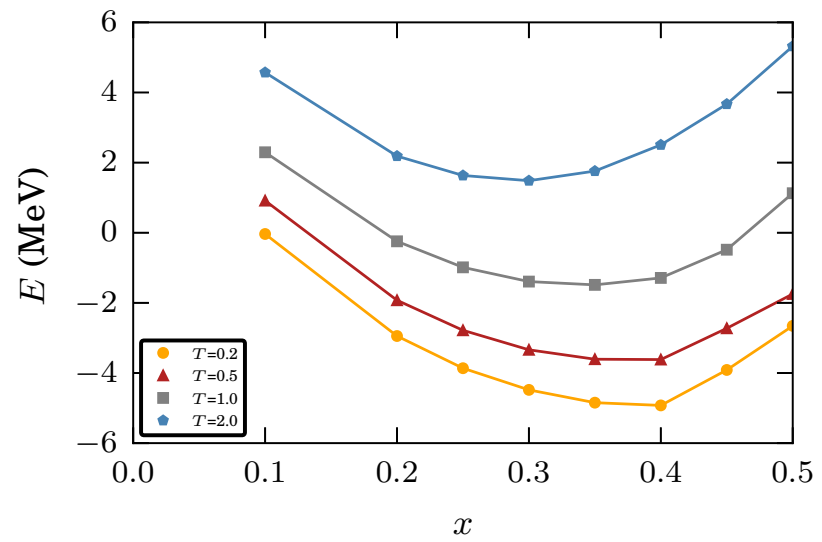

(a) $\rho=0.04$

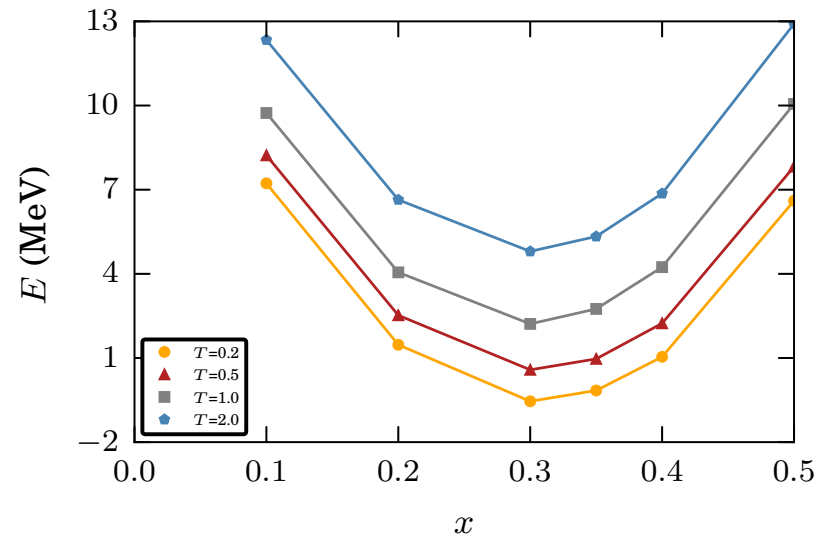

(b) $\rho=0.085$

FIG. 10. (color online) Internal energy per nucleon for neutron star matter systems with $N=4000$ nucleons. All the curves correspond to the New Medium model (see Section II). (a) Mean density $\rho=0.04$. (b) Mean density $\rho=0.085$.

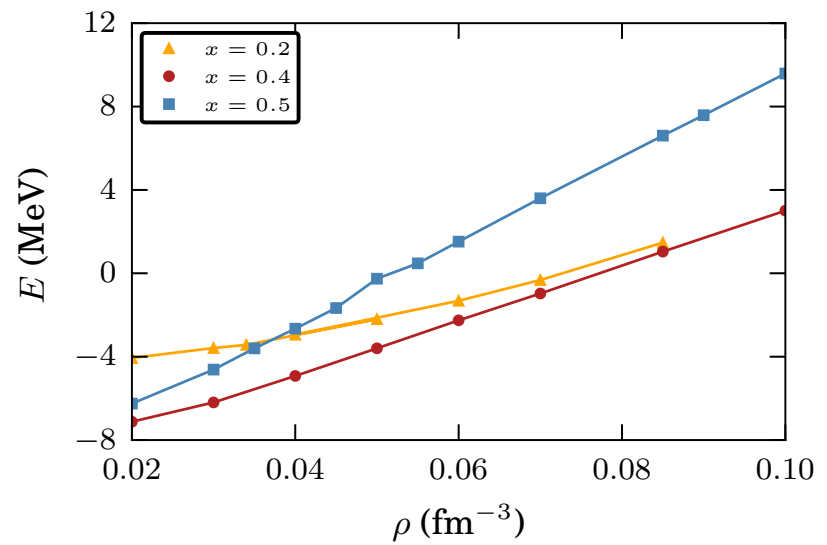

(a) $T=0.2$

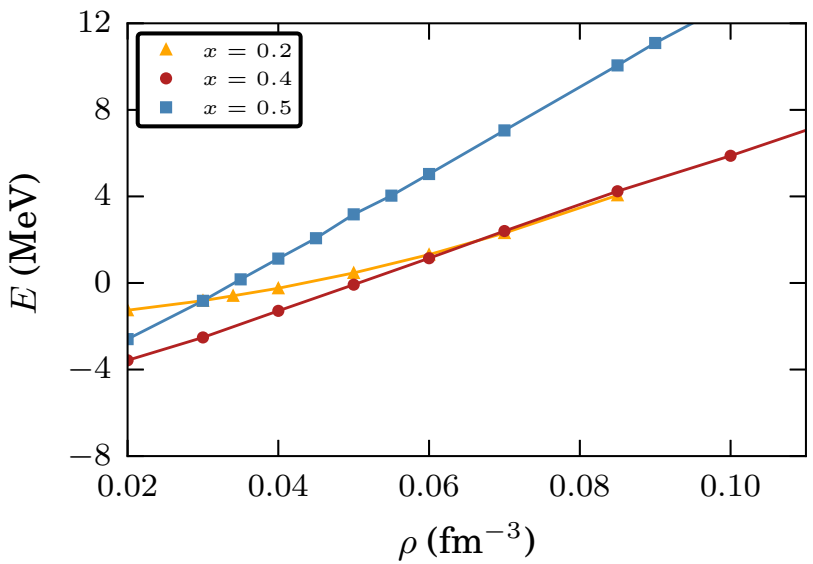

(b) $T=1.0$

FIG. 11. (color online) Internal energy per nucleon for neutron star matter systems of $N=4000$ nucleons at three proton fractions and interacting through the New Medium model. (a) Temperature $T=0.2 \mathrm{MeV}$. (b) Temperature $T=1.0 \mathrm{MeV}$.

Further details can be understood by looking at the radial distribution function. Fig. 15 shows the $g(r)$ for two representative proton fractions at opposed densities. Notice from Fig. $15 \mathrm{~b}$ that no qualitative differences can be distinguished between the $x=0.2$ and the $x=0.4$ situations. Neither can be distinguished between these and the symmetric case shown in Figure 8 (for $\rho=0.085)$, except that the peaks appear somewhat sharper. Thus, the overall topology does not experience relevant changes for decreasing proton fractions at the highest explored density.

Figure 13 shows three snapshots for the protons alone at $\rho=0.085 \mathrm{fm}^{-3}$. The hollow spaces in there actually correspond to regions occupied by neutrons (not represented for the sake of clarity). It can be seen that the number of hollow regions (that is, the neutron regions) increases for decreasing fractions, while inside the "proton regions" no relevant changes can be noticed. This explains why the overall $g(r)$ does not exhibit qualitative changes in Fig. 15b, while Fig. 10b shows the "right-branch" pattern.

The radial distribution for the lower density $\rho=0.02 \mathrm{fm}^{-3}$ is qualitatively different from those at $\rho=0.085 \mathrm{fm}^{-3}$, as can be seen in Fig. 15a. The profiles appear sharply concentrated along the first few femtometers, while they gradually vanish towards $r \sim 10 \mathrm{fm}$. Both profiles in Fig. 15a correspond to gnocchi-like structures, similar to the one shown in 


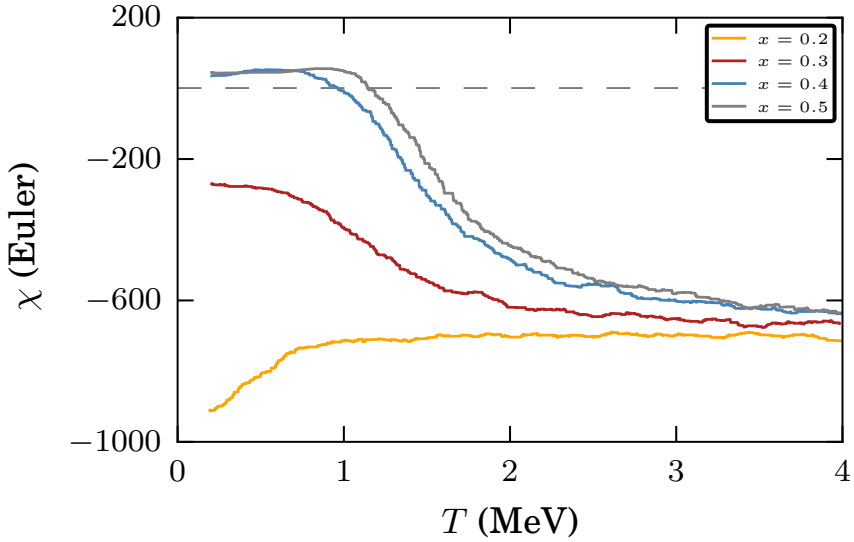

(a) $\rho=0.04$

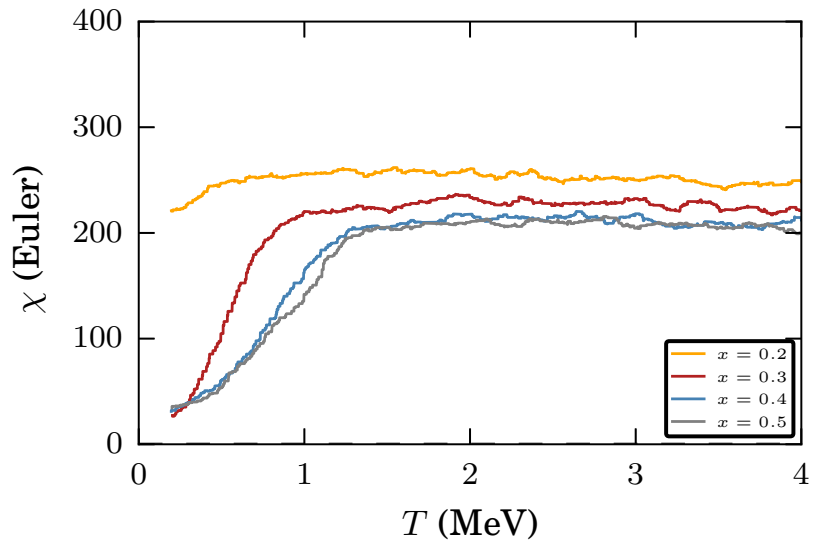

(b) $\rho=0.085$

FIG. 12. (color online) The Euler functional $\chi$ as a function of temperature for non-symmetric New Medium model. The corresponding proton fractions $x$ are indicated in the inset. The total number of nucleons is $N=4000$. The binning distance is $d=2.35 \mathrm{fm}$. The horizontal line corresponds to the null level. (a) The system mean density is $\rho=0.04 \mathrm{fm}^{-3}$. (b) The system mean density is $\rho=0.085 \mathrm{fm}^{-3}$. The data has been smoothed with a moving average procedure.

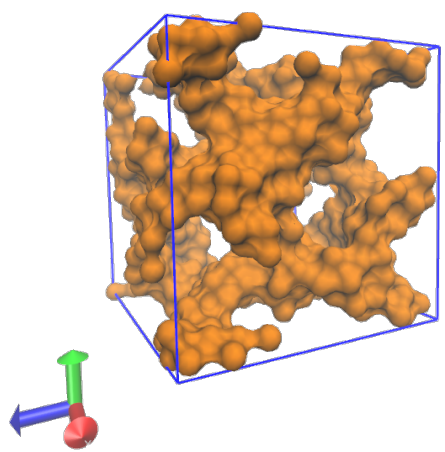

(a) $x=0.2$

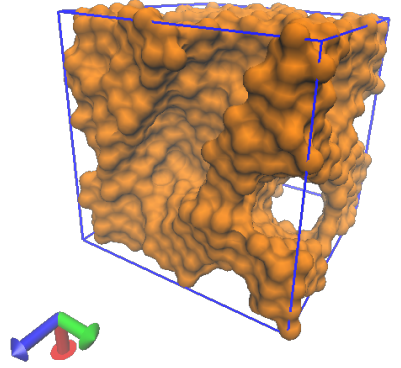

(b) $x=0.4$

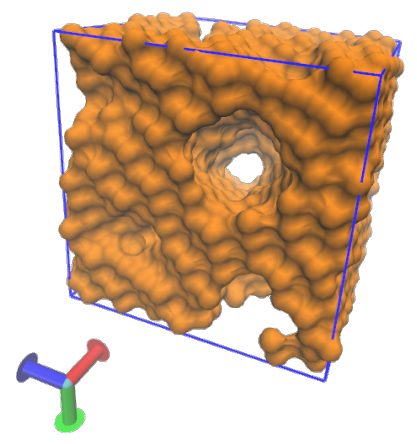

(c) $x=0.5$

FIG. 13. (color online) Surface representation for the protons at the indicated proton fractions. The snapshot was taken at $T=0.2 \mathrm{MeV}$ and $\rho=0.085 \mathrm{fm}^{-3}$ for a system of 4000 nucleons under periodic boundary conditions interacting through the New Medium Model plus the screened Coulomb potential.

Fig. 9a for symmetric matter (not shown). Notice, however, that as the proton fraction diminishes, the profiles become somewhat smoother (see Fig. 15a and Fig. 8).

Figure 16 splits Figure 15a into the corresponding profiles for protons and neutrons (see caption for details). It becomes clear from the comparison between Figures 16a and $16 \mathrm{~b}$ that the smoothening process in Figure 15a for decreasing proton fractions is related to the $g(r)$ distribution of the species, within the gnocchi structure (say, $r \leq 6 \mathrm{fm}$ ). Specifically, the proton-neutron (P-N) and neutron-neutron (N-N) distributions smoothen when the fraction $x$ diminishes. Notice, however, that the proton-proton distribution $g(r)$ sharpens around $3 \mathrm{fm}$. But this does not balance the P-N and N-N smoothening because of the reduced number of protons for $x=2$.

We may further examine the simulation cell for the 


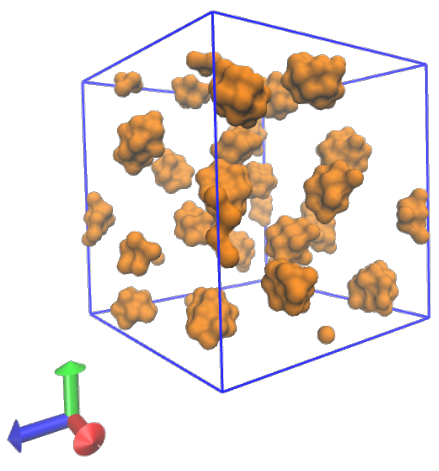

(a) $x=0.1$

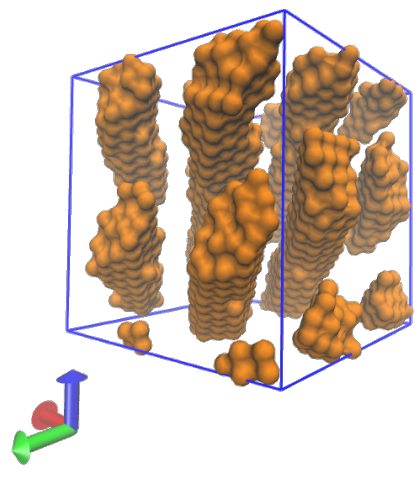

(d) $x=0.4$

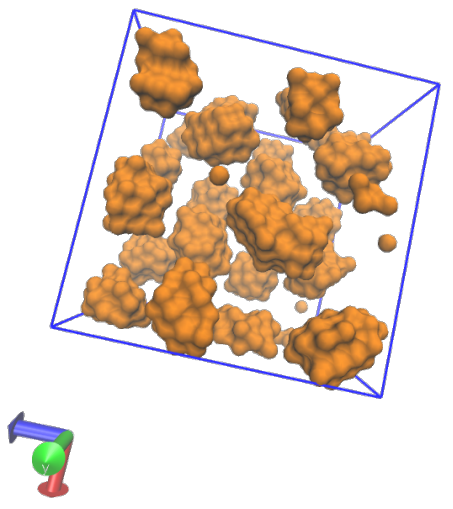

(b) $x=0.2$

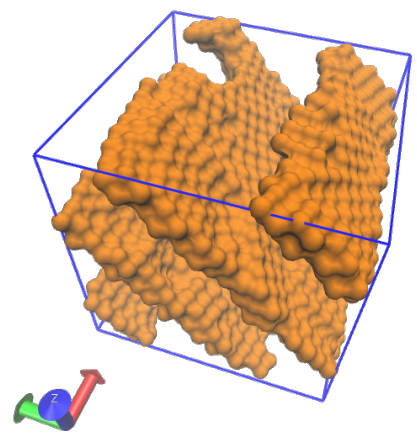

(e) $x=0.45$

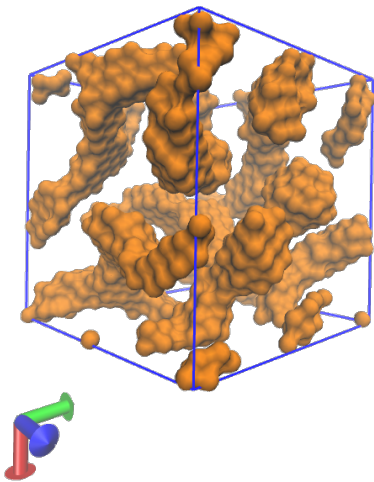

(c) $x=0.3$

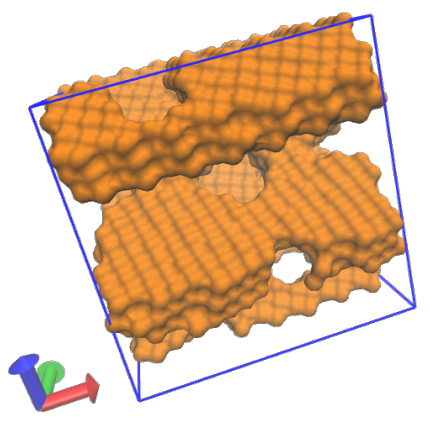

(f) $x=0.5$

FIG. 14. (color online) Surface representation for the protons at the indicated fractions. The snapshot was taken at $T=0.2$ and $\rho=0.04$ for a system of 4000 nucleons.

intermediate density $\rho=0.04 \mathrm{fm}^{-3}$. Fig. 14 exhibits the corresponding proton structures (neutron not shown) for a sequence of fractions $x<0.5$. We can see that as the proton fraction decreases, the "pasta" splits into smaller pieces. The "pasta" structures for nearly symmetric neutron star matter $(x \simeq 0.5)$ presents as lasagna-like structures, while the low proton fraction scenario $(x<0.3)$ forms gnocchi-like structures. The spaghetti-like structures appear in between $(x \simeq 0.4)$.

From the comparison between Fig. 10 and Fig. 14 we confirm that the changing topology of the system is due to the $\cup$-shape pattern for the internal energy as a function of $x$. The spaghetti-like structure achieves the minimum energy. The lasagnas and the gnocchis correspond to higher energies on either branch of the $U$-shape.

In summary, two major effects appear when departing from symmetry. A topological re-arrangement of the pasta structures occurs, attaining some kind of fragmentation as the proton fraction diminishes. The lowest explored proton fraction attains the gnocchi structure, but, the gnocchis themselves experience (inner) topological changes during its formation process. The resulting energy level surpasses the one at $x \sim 0.3$ in agreement with the observations made in reference to the energy $\cup$-shape dependence on $x$. 


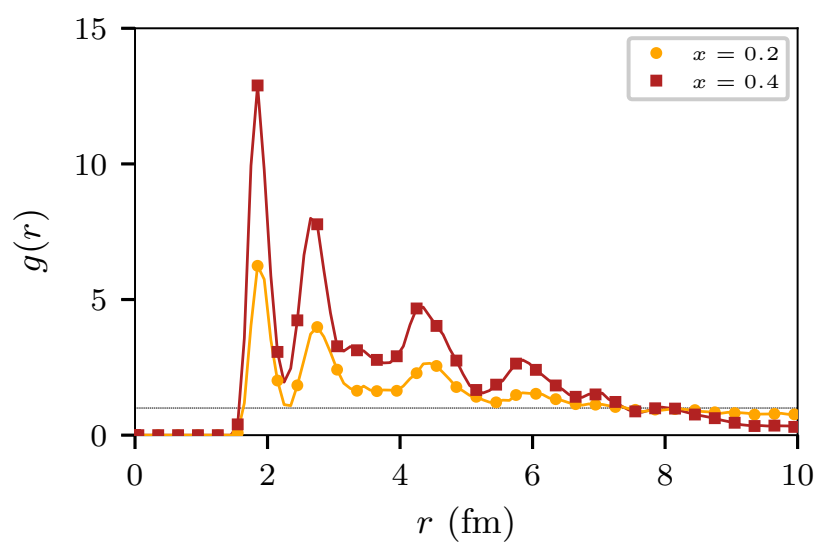

(a) $\rho=0.02$

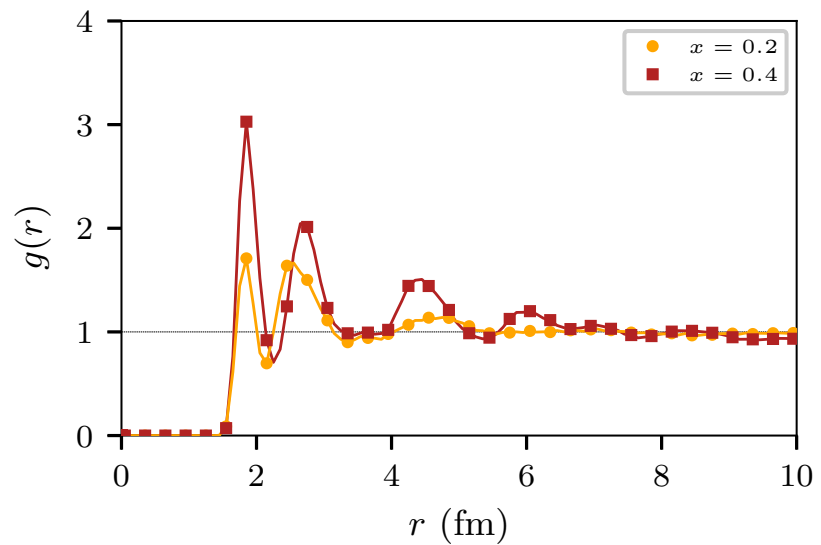

(b) $\rho=0.085$

FIG. 15. (color online) Radial distribution function $g(r)$ for nucleons corresponding to non-symmetric neutron star matter systems with $N=4000$ nucleons at $T=0.2 \mathrm{MeV}$. The system proton fraction is indicated in the inset. The black horizontal line is a view guide for $g(r)=1$. All the curves correspond to the New Medium model (see Section II). (a) Density $\rho=0.02$. (b) Density $\rho=0.085$.

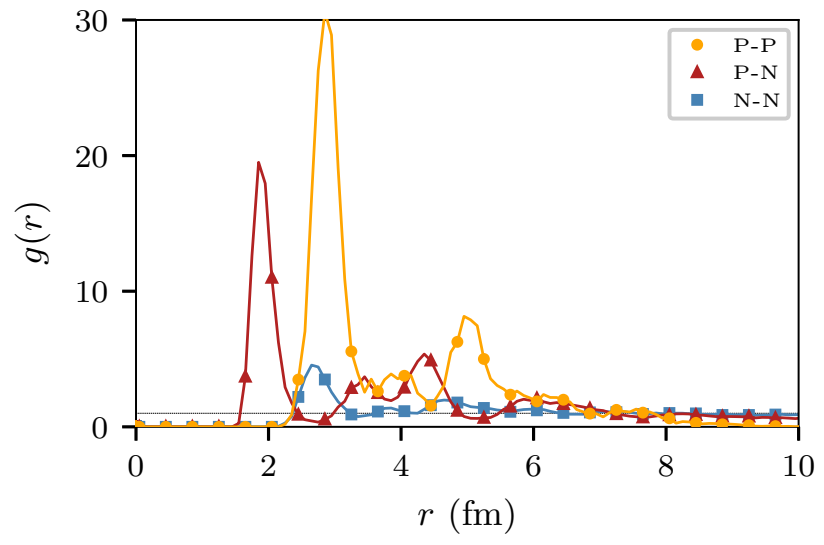

(a) $x=0.2$

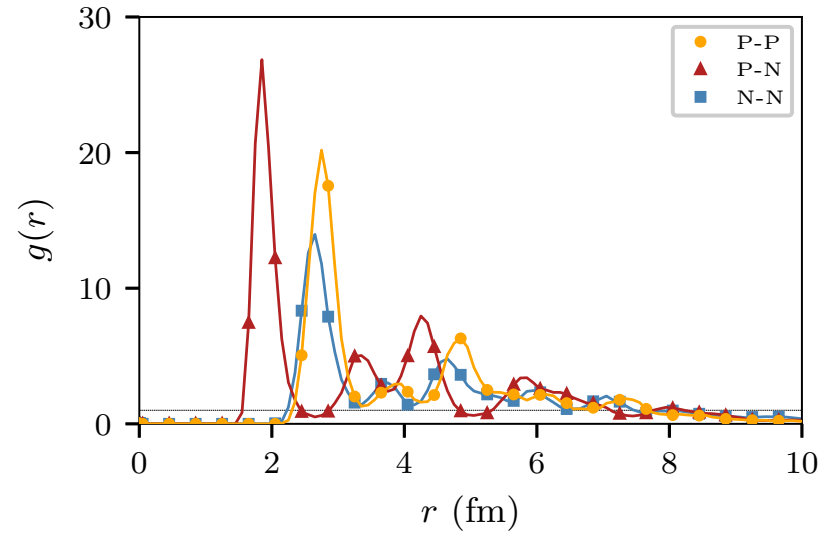

(b) $x=0.4$

FIG. 16. (color online) Radial distribution function $g(r)$ for nucleons corresponding to non-symmetric neutron star matter at $T=0.2 \mathrm{MeV}, \rho=0.01 \mathrm{fm}^{-3}$, and (a) $x=0.2$ and (b) $x=0.4$. The total number of nucleons is $N=4000$. In the inset "P-P" stands for proton-proton distances only, "P-N" corresponds to proton-neutron distances only, and "N-N" means neutron-neutron only.

\section{The Symmetry energy}

We pointed out in Sections III B 1 and III B 2 that topological changes taking place for decreasing proton fractions are indeed related to the $\cup$-shape dependence of the energy on $x$ shown in Figure 10. These patterns also carry information on the symmetry energy contribution to the equation of state; in this section we calculate $E_{\text {syn }}$ according to the procedure detailed in Section IIIC (and Appendix A).

Fig. 17 shows the fittings for the internal energy as a function of the proton fraction $x$. The curves represent the expected internal energy, according to Equation (6), although the term of $\mathcal{O}\left(\alpha^{4}\right)$ was neglected for simplicity (see Appendix A for details). Data appears to fit quite well into this quadratic profile. However, the low temperature curves shown in Fig. 17a exhibit some kind of departure from data at $x \sim 0.4$. This is somewhat an $\mathcal{O}\left(\alpha^{4}\right)$ discrepancy since a noticeable improvement can be obtained if an $\alpha^{4}$ term is added into the fitting procedure (not shown).

The fittings for the highest explored density (see 


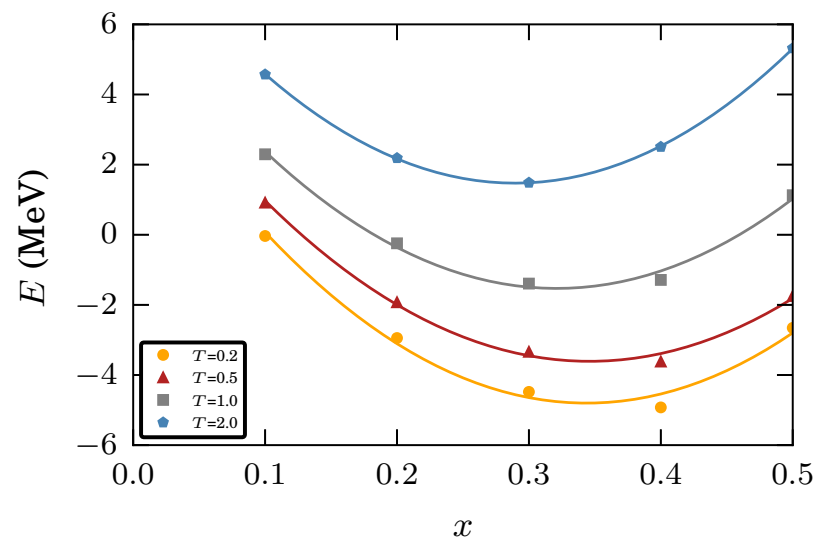

(a) $\rho=0.04$

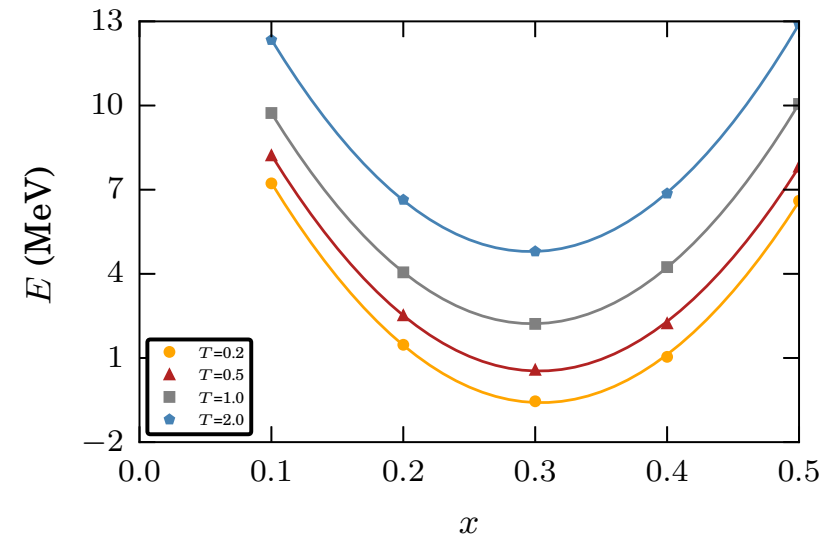

(b) $\rho=0.085$

FIG. 17. (color online) Internal energy per nucleon for neutron star matter at the indicated densities for systems with $N=4000$ nucleons interacting through the New Medium model. The rounded symbols correspond to CMD data, and the lines correspond to the estimated values after the two-step fitting procedure mentioned in Section IIIC and Appendix A

Fig. 17b match better the CMD data than the fittings at $\rho=0.04$ (Fig. 17a). Recall that the former correspond to more compact proton structures (see Fig. 13) than the latter (see Fig. 14). Thus, as already noticed in Section IIIB 1 and and IIIB 2 more "fragmented" structures (say, spaghettis instead of lasagnas) flattens the right-branch of the U-shape pattern in Fig. 17a, departing from a seemingly quadratic profile. The fittings discrepancy expresses this issue.

We further proceed to compute the $E_{\text {sym }}$ for neutron star matter (within the explored range). Fig. 18b shows the computed $E_{\text {sym }}$ for two different densities. The corresponding $E_{\text {sym }}$ for nuclear matter (Medium Model) appearing in Ref. [30] has also been included for comparison. Notice that the $E_{\text {sym }}$ levels for nuclear matter and neutron star matter are essentially the same above $T \sim 2 \mathrm{MeV}$, but differ for lower temperatures. The upper regime (above $2 \mathrm{MeV}$ ) confirms that the $E_{\text {sym }}$ has been computed accurately, since either the internal energy in Fig. 17a and the $E_{\text {sym }}$ match the expected values.

It can be seen in Fig. $18 \mathrm{~b}$ a smooth change in the profile slopes for temperatures below $1.5 \mathrm{MeV}$. This is (roughly) in agreement with the patterns shown in Fig. 18a for the nuclear matter (Medium) model, although the slopes' signs are different. The neutron star matter profiles appear (qualitatively) inverted with respect to the nuclear matter profiles (say, the slopes present opposite signs).

The estimation with the $\mathcal{O}\left(\alpha^{4}\right)$ term fits better the CMD data exhibited in Fig. 11 for $x \sim 0.4$ and for the lowest explored temperature (not shown). However, the $E_{\text {sym }}$ level at higher temperatures (above $1.5 \mathrm{MeV}$ ) spreads out, not allowing a precise estimation of the corresponding energy level. Thus, the quadratic order estimations shown in Fig. 18 are somehow more practical.

The major result from the above observations is that the $E_{\text {sym }}$ experiences a change in its slope at the pasta regime, driving the energy level to lower values for decreasing temperatures (below $1.5 \mathrm{MeV}$ ). This is a somewhat opposite behavior with respect to the known results for the nuclear matter model (see Ref 30]). Furthermore, this behavior holds for either fittings of order $\alpha^{2}$ and $\alpha^{4}$.

A second result can be outlined from the widely explored range of the proton fraction. The "left-branches" appearing in Fig. 17 handle somehow the $E_{\text {sym }}$ level at the pasta regime (below $2 \mathrm{MeV}$ ). But, as mentioned in Section IIIB 2, this "left-sided" behavior accomplishes topological changes within the (fragmented) pasta structures at low densities (within the explored range). Thus, the re-arrangements in the (fragmented) pasta structures (say, gnocchi-like or spaghetti-like) themselves are able to switch the $E_{\text {sym }}$ level up or down with respect to the level above $2 \mathrm{MeV}$. In brief, the presence of pasta influences the behavior of the $E_{\text {sym }}$ both for NM and NSM.

\section{CONCLUSIONS}

In this article we have studied the behavior of Neutron Star matter (according to the CMD approach). At rather low temperatures $(0.2 \mathrm{MeV} \leq T \leq 4 \mathrm{MeV})$ and densities in the range of $0.02 \mathrm{fm}^{-1}$ to $0.085 \mathrm{fm}^{-1}$ for isospin symmetric $(x=0.5)$ and non-symmetric $(x=0.1-0.4)$. We have investigated the formation of "pastas", the emergence of phase transitions and 


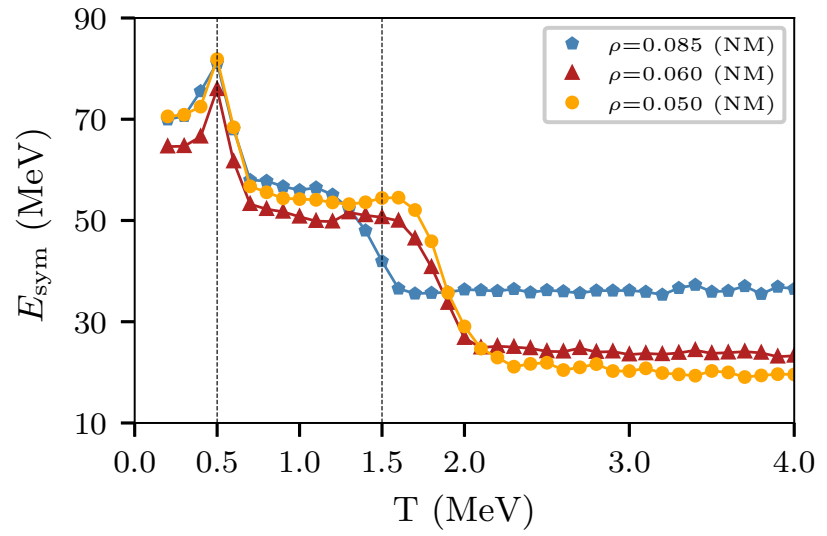

(a) nuclear matter

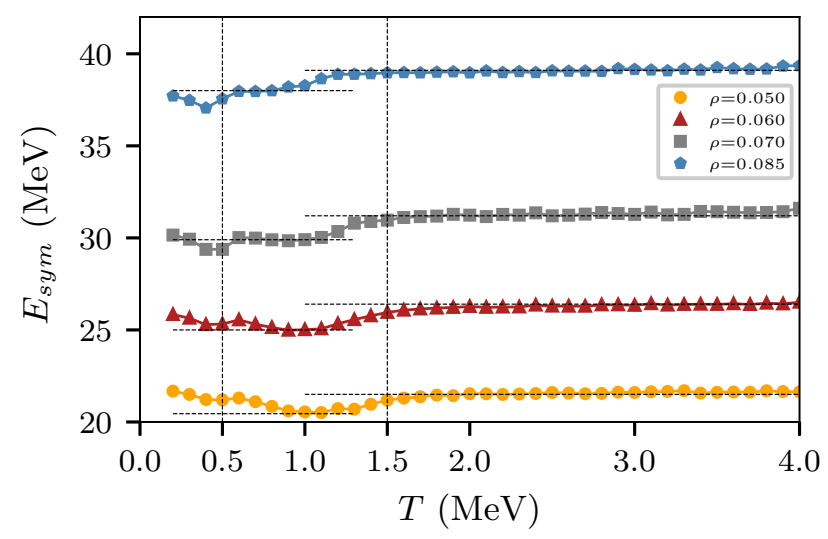

(b) neutron star matter

FIG. 18. (color online) Symmetry energy as a function of the temperature for the densities indicated in the insert in $\mathrm{fm}^{-3}$. (a) Symmetry energy for nuclear matter (Pandharipande Medium Model) as a function of the temperature (extracted from Ref. [30]). The vertical dashed lines are a guide to the eye. (b) Symmetry energy for $N=4000$ nucleons embedded in an electron cloud, as explained in Section II The fitting procedures are detailed in Section III C and Appendix A The horizontal and vertical dashed lines are a guide to the eye.

the behavior of the symmetry energy $\left(E_{\text {sym }}\right)$. Using different techniques of molecular dynamics we studied "infinite systems" using primordial cell of 4000 nucleons and periodic boundary conditions.

We calculated the caloric curve and found that as temperature was lowered from $4 \mathrm{MeV}$ at a given point (irrespective of the value of $x$ in the studied range) a clear change of the slope takes place, signaling the break down of the homogeneous situation as the system undergoes the topological phase transition. If the temperature is further lowered the signal of the first order (liquid to solid) phase transition is clearly seen. At variance with the case of the nuclear matter, the caloric curves (CC) for the different values of $\rho$ remain parallel.

The behavior of the system was also analyzed using the Euler functional $\chi$. For temperatures above $T \simeq 1 \mathrm{MeV}, \chi$ increases with density. If the densities are $\rho=0.05-0.06 \mathrm{fm}^{-1}, \chi$ goes from negative values (tunnel dominated configurations) to positive ones (configurations dominated by cavities).

All these phase changes that were detected as the temperature was lowered are reflected in the behavior of the symmetry energy. It is clearly seen that for temperatures above $2 \mathrm{MeV}$, the symmetry energy calculated according to the method explained in the appendix remains basically constant. On the other hand, for temperatures around the values for which we have changes in the slope of the caloric curves the symmetry energy also displays changes, as was stated for the case of nuclear matter.
In conclusion, classical molecular dynamics simulations show the formation of "pastas" in the isospin symmetric and non-symmetric nuclear star matter. The behavior of $E_{\text {sym }}$, together with the two phase transitions are an indication of interesting phenomena that are present in the neutron star crust and supernova explosions.

\section{ACKNOWLEDGMENTS}

Part of this study was financed by FONCyT (Fondo para la Investigación Científica y Tecnológica) and InterAmerican Development Bank (IDB), Grant Number PICT 1692 (2013).

\section{Appendix A: The symmetry energy fitting procedure}

The fitting procedure outlined in Section IIB 2 corresponds to a two step-fitting method. The coefficients $E_{i}$ $(i=0 \ldots 3)$ from Eq. (5) are computed at the first stage of the procedure. These coefficients, however, depend on the fraction $\alpha$, although not directly on the fraction $x$ (recall that $\alpha=1-2 x$ ). A more suitable (low order) expression for the $E_{i}$ 's instead of Eq. (6) is

$$
\begin{aligned}
E_{i}(T, x) & =E_{i 0}+E_{i 2} \overbrace{\left(1-4 x+4 x^{2}\right)}^{\alpha^{2}}+\mathcal{O}\left(\alpha^{4}\right) \\
& \simeq\left(E_{i 0}+E_{i 2}\right)-4 E_{i 2} x+4 E_{i 2} x^{2}
\end{aligned}
$$

The above expression corresponds to the lowest non-trivial expansion $E_{i}(T, x) \simeq \tilde{E}_{i 0}+\tilde{E}_{i 1} x+\tilde{E}_{i 2} x^{2}$. 
Thus, the $\tilde{E}_{i j}$ 's $(j=0,1,2)$ are related to the $E_{i 0}, E_{i 2}$ coefficients according to the following matrix relation

$$
\left(\begin{array}{cc}
1 & 1 \\
0 & -4 \\
0 & 4
\end{array}\right)\left(\begin{array}{c}
E_{i 0} \\
E_{i 2}
\end{array}\right)=\left(\begin{array}{c}
\tilde{E}_{i 0} \\
\tilde{E}_{i 1} \\
\tilde{E}_{i 2}
\end{array}\right)
$$

Notice that $\tilde{E}_{i 0}, \tilde{E}_{i 1}, \tilde{E}_{i 2}$ actually correspond to the fitting parameters at the second stage of the procedure outlined in Section IIB2 (that is, after the $E_{i}$ 's were obtained). The $E_{i 0}$ and $E_{i 2}$ parameters, though, are now computed indirectly from the overdetermined system A2 . The best estimates for $E_{i 0}$ and $E_{i 2}$ that we can think about correspond to the least squares estimation. Thus, the estimate for $E_{i 2}$ gives

$$
E_{i 2}=\frac{\tilde{E}_{i 2}-\tilde{E}_{i 1}}{8} \pm \frac{1}{\sqrt{2}} \frac{\tilde{E}_{i 2}-\tilde{E}_{i 1}}{8}
$$

where the first term on the right corresponds to the estimated (least squares) mean value, while the second term represents the standard deviation.

The resulting estimate for $E_{i 2}$ in A3 is actually the mid-value between the (incompatible) solutions $-\tilde{E}_{i 1} / 4$ and $\tilde{E}_{i 2} / 4$ that can be obtained (independently) from the second and third rows in $\mathrm{A} 2$, respectively. Thus, the estimation A3 may be envisaged as the resultant for equally weighting the linear and the quadratic terms in Eq. A1). This is the key consequence of introducing the substitution $\alpha \rightarrow x$ in Eq. A1.

Our numerical computations for $E_{i 2}$ at the examined temperatures and densities indicated in Section III C are detailed in Table III.

\begin{tabular}{crrrr}
\hline \hline$T$ & $E_{02}$ & $E_{12}$ & $E_{22}$ & $E_{32}$ \\
\hline 0.2 & -9.472 & 976 & -10080 & 60305 \\
0.5 & -35.721 & 2249 & -30633 & 168400 \\
1.0 & 0.745 & 408 & -1137 & 18048 \\
2.0 & 3.401 & 239 & 2985 & -10323 \\
\hline \hline
\end{tabular}

TABLE III. Least squares estimates according to Eq. A3. The standard deviations may be obtained easily from these estimates and Eq. (A3).
[1] L.F. Roberts, G. Shen, V. Cirigliano, J.A. Pons, S. Reddy, and S.E. Woosley Phys. Rev. Lett. 108, 061103,2012

[2] C.O. Dorso, P.A. Giménez Molinelli and J.A. López, in "Neutron Star Crust", Eds. C.A. Bertulani and J. Piekarewicz, Nova Science Publishers, ISBN 9781620819029 (2012).

[3] K. Hagel, J.B. Natowitz and G. Röpke, Eur. Phys. J. A 50, 39 (2014)

[4] S. Kowalski, et al., Phys. Rev. C 75, 014601 (2007).

[5] R. Wada, et al., Phys. Rev. C 85, 064618 (2012).

[6] C.O. Dorso, P.A. Giménez Molinelli and J.A. López, Phys. Rev. C86, 055805 (2012).

[7] B.A. Li, L.W. Chen and C.M. Ko, Phys. Rep. 464, 113 (2008).

[8] D.G. Ravenhall, C. J. Pethick and J. R. Wilson, Phys. Rev. Lett. 50, 2066 (1983).

[9] M. Hashimoto, H. Seki and M. Yamada, Prog. Theor. Phys. 71, 320 (1984).

[10] D. Page, J. M. Lattimer, M. Prakash and A. W. Steiner, Astrophys. J. Supp. 155, 623 (2004)

[11] R. D. Williams and S. E. Koonin, Nucl. Phys. A435, 844 (1985).

[12] P.N. Alcain, P.A. Giménez Molinelli and C.O. Dorso, Phys. Rev. C90, 065803 (2014).

[13] J. A. López, E. Ramírez-Homs, R. González, and R. Ravelo Phys. Rev. C89, 024611 (2014).

[14] P.N. Alcain and C.O. Dorso, Nuclear Phys. A 961, 183 (2017).

[15] T. Maruyama, K. Niita, K. Oyamatsu, T. Maruyama, S. Chiba and A. Iwamoto, Phys. Rev. C57, 655 (1998).
[16] T. Kido, Toshiki Maruyama, K. Niita and S. Chiba, Nucl. Phys. A663-664, 877 (2000).

[17] G. Watanabe, K. Sato, K. Yasuoka and T. Ebisuzaki, Phys. Rev. C66, 012801 (2002).

[18] Watanabe et al. Phys. Rev. C 68, 035806 (2003)

[19] Schneider et al. Phys. Rev. C 88, 065807 (2013)

[20] Caplan et al. Rev. Mod. Phys. 89, 041002 (2017)

[21] Schuetrumpf et al. Phys. Rev. C 87, 055805 (2013)

[22] P.N. Alcain and C.O. Dorso, Phys. Rev. C97, 015803 (2018).

[23] A. Vicentini, G. Jacucci and V.R. Pandharipande, Phys. Rev. C31, 1783 (1985); R. J. Lenk and V. R. Pandharipande, Phys. Rev. C34, 177 (1986); R.J. Lenk, T.J. Schlagel and V. R. Pandharipande, Phys. Rev. C42,372 (1990).

[24] S. Plimpton, J. Comp. Phys., 117, 1-19 (1995).

[25] C.J. Horowitz, M.A. Pérez-García, J. Carriere, D.K. Berry, and J. Piekarewicz, Phys. Rev. C70, 065806 (2004).

[26] T. Maruyama, K. Niita, K. Oyamatsu, T. Maruyama, S. Chiba and A. Iwamoto, Phys. Rev. C57, 655 (1998).

[27] K. Michielsen and H. De Raedt, Phys. Rep. 347, 461 (2001).

[28] P.N. Alcain, P.A. Giménez Molinelli, J.I. Nichols and C.O. Dorso, Phys. Rev. C89, 055801 (2014).

[29] J.A. López and S. Terrazas Porras, Nuc. Phys. A957, 312 (2017).

[30] C.O. Dorso, G.A. Frank and J.A. López, Nuc. Phys. A978, 35 (2018). 